\title{
Self-Initiation Mechanism in Spontaneous Thermal Polymerization of Ethyl and n-Butyl Acrylate: A Theoretical Study
}

\author{
Sriraj Srinivasan, ${ }^{\dagger}$ Myung Won Lee, ${ }^{\ddagger}$ Michael C. Grady, ${ }^{\S}$ Masoud Soroush, ${ }^{\dagger}$ and \\ Andrew M. Rappe*,: \\ Department of Chemical and Biological Engineering, Drexel University, Philadelphia, Pennsylvania, Makineni \\ Theoretical Laboratories, Department of Chemistry, University of Pennsylvania, Philadelphia, Pennsylvania, \\ and Experimental Station, DuPont, Wilimington, Delaware
}

Received: March 27, 2010; Revised Manuscript Received: May 16, 2010

\begin{abstract}
In this study, the mechanism of self-initiation in spontaneous thermal polymerization of ethyl and n-butyl acrylate is explored using first-principles calculations. Density functional theory (with B3LYP functional and 6-31G* basis set) was used to study [4 +2$]$ and $[2+2]$ cycloaddition reactions on the singlet and triplet potential energy surfaces. Diels-Alder (DA) dimers of ethyl acrylate [6-ethoxy-2-ethoxycarbonyl-3,4-dihydro$2 H$-pyran (EDP)] and of n-butyl acrylate [6-butoxy-2-butoxycarbonyl-3,4-dihydro-2H-pyran (BDP)] were found to form on the singlet surface via the concerted pathway proposed by Mayo. The formation of diethyl cyclobutane-1,2-dicarboxylate (DECD) and dibutyl cyclobutane-1,2-dicarboxylate (DBCD) via a nonconcerted pathway was identified on the singlet surface of ethyl and n-butyl acrylate, respectively. The presence of a diradical transition state for the formation of the DECD and DBCD was predicted. Triplet potential energy surfaces for the formation of diradical dimer of ethyl and n-butyl acrylate were computed, and the presence of a triplet diradical intermediate was identified for each of the monomers. A low energy monoradical generation mechanism was identified to be involving hydrogen abstraction by a third acrylate monomer from the triplet diradical species. The molecular structure of the computed monoradical species was found to correlate with chain initiating species of the dominant series of peaks in previously reported electrospray ionization-Fourier transform mass spectra of spontaneously polymerized samples of ethyl and n-butyl acrylate. In view of these observations, it is concluded that this self-initiation mechanism is most likely the initiating mechanism in spontaneous thermal polymerization of alkyl acrylates.
\end{abstract}

\section{Introduction}

Stringent environmental regulations have driven a change in the basic nature of acrylic resins used in automobile coatings. ${ }^{1,2}$ While the conventional process involved the use of low temperature and low solids to produce high molecular weight resins, newer processes have used high temperatures $\left(>100{ }^{\circ} \mathrm{C}\right)$ and high solids to generate low molecular weight resins. ${ }^{3-6}$ At these high temperatures, the occurrence of chain transfer reactions such as backbiting (intra- or intermolecular hydrogen transfer to monomer or solvent) and subsequent $\beta$-scission reactions, as well as depropagation, has been reported. ${ }^{4-6}$ Grady et $\mathrm{al}^{7}$ showed, for the first time, that, at high temperatures, spontaneous polymerization of alkyl acrylates was possible in the absence of any known added extraneous initiating species. Previous studies ${ }^{8,9}$ show that backbiting and $\beta$-scission reactions can generate chain-initiating species ( $\beta$-scission radicals) and cause the production of acrylic resins with a narrow polydispersity index (1.4-1.6) after prolonged polymerization ( $\approx 24 \mathrm{~h}$ ). However, the initiating $\beta$-scission radical forms during chain propagation, which suggests that these radicals cannot be the true initiating species in the spontaneous polymerization of alkyl acrylates. Recently, we postulated and computationally verified ${ }^{10}$ that self-initiation of methyl acrylate is likely initiating mechanism in spontaneous thermal polymerization of methyl

\footnotetext{
* Corresponding author. E-mail: rappe@sas.upenn.edu.

${ }^{\dagger}$ Drexel University.

* University of Pennsylvania.

$\S$ DuPont.
}

acrylate. Analysis of the spontaneously polymerized samples of methyl acrylate using matrix assisted laser desorptionionization (MALDI) mass spectrometry provided laboratory evidence pointing to chain initiation by the initiating species generated via the postulated and computationally verified mechanism. ${ }^{11}$ As ethyl and n-butyl acrylate polymers are more widely used in comparison to methyl acrylate in industrial coating formulations, it becomes important to identify the mechanism of initiation in spontaneous thermal polymerization of ethyl and n-butyl acrylate. Whether the rates of initiation can be correlated to the size of the end-substituent group in the spontaneous polymerization of n-butyl, ethyl, and methyl acrylate needs to be understood. As of yet, no computational study using quantum chemical calculations has been carried out to elucidate the mechanism of initiation and the initiating species in spontaneous polymerization of ethyl and n-butyl acrylate. Also, this study looks to provide further evidence to the existence of self-initiation in polymerization of alkyl acrylates.

Spontaneous polymerization of styrene and methyl methacrylate (MMA) has been extensively studied. ${ }^{12-19}$ These monomers were reported to self-initiate in the absence of any known added external initiators or catalysts. ${ }^{13,14}$ The Mayo mechanism $^{14}$ of self-initiation postulates that two monomers combine to form a Diels-Alder (DA) adduct, which then loses a hydrogen to a third monomer (molecular assisted homolysis) to form two monoradical species (Figure 1). The evidence of the occurrence of the Mayo mechanism in spontaneous thermal polymerization of styrene has been provided via laboratory 

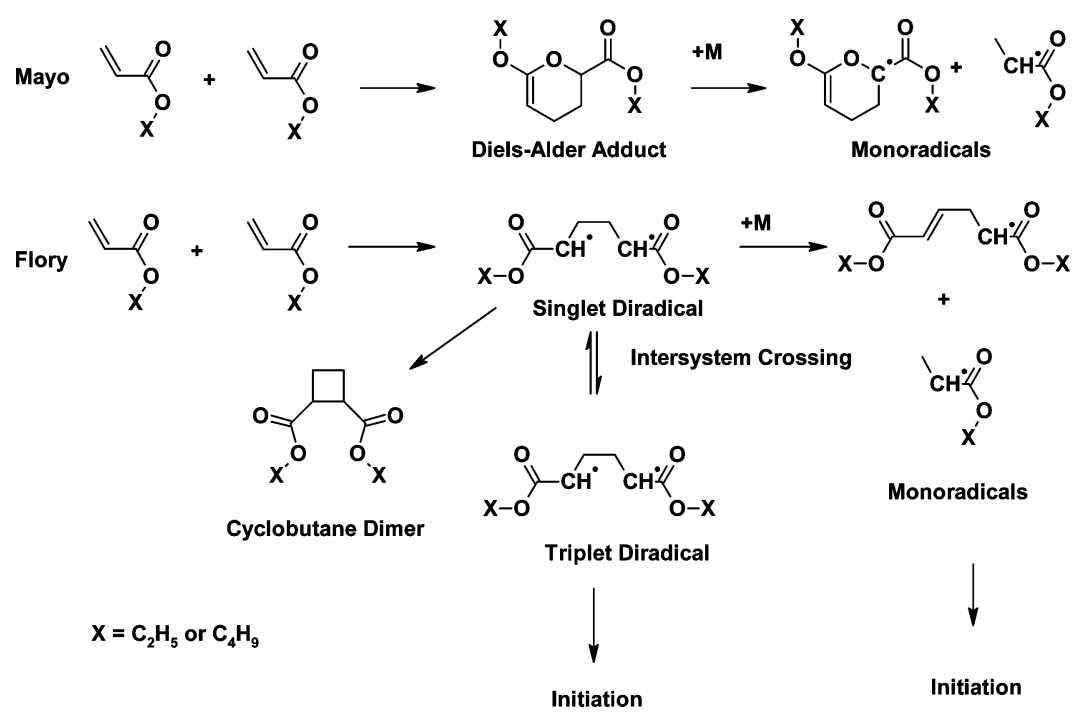

Figure 1. Flory and Mayo mechanisms of self-initiation for ethyl acrylate and n-butyl acrylate.

experiments, ${ }^{15-18}$ and theoretical studies using density functional calculations. ${ }^{20}$ By contrast, Flory's mechanism ${ }^{13}$ of self-initiation proposes two monomers to combine and form a diradical, which either undergoes ring closure to form a cyclobutane derivative (reversible reaction) or undergoes hydrogen transfer or abstraction with a third monomer to form monoradical species. However, whether the diradical in its ground (singlet) state can undergo hydrogen transfer or abstraction reactions is questionable. Pryor and Laswell ${ }^{18}$ postulated that the diradical species, probably in a triplet (excited) state, is the one that generates monoradical species for initiating polymerization in nonstyrenic monomers. The absence of Diels-Alder adducts and the involvement of diradical species in initiation of spontaneous thermal polymerization of MMA, which supports the postulate of Pryor and Lasswell, ${ }^{18}$ have been reported. ${ }^{19}$ Our recent computational study shows the inability of the Diels-Alder adducts to form monoradical species, and the necessity of a triplet diradical intermediate to generate initiating species for spontaneous thermal polymerization of methyl acrylate. ${ }^{10}$

Salem and Rowland ${ }^{21}$ and others ${ }^{22-27}$ have reported the occurrence of diradical crossover from singlet to triplet state, the rules for such crossover, and the possible mechanisms for such intersystem crossing. The spin-orbit coupling mechanism ${ }^{21}$ for intersystem crossing from diradical singlet to triplet state has been shown to occur in various species, in particular, those similar in size and structure to ethyl and n-butyl acrylate. An important requirement to facilitate crossover is the existence of a dense manifold of vibrational states in the singlet-triplet system. $^{22}$ This increases the probability for overlap of singlet and triplet vibrational states and subsequent intersystem crossing. Previous studies indicate that collision between diradicals and inert solvent, monomer or gas molecules at high temperatures can increase the rate of crossover. ${ }^{23-25}$

Previous computational studies show that the transition state geometries and energy barriers calculated in the gas phase and in inert solvents such as toluene and benzene using solvent continuum models ${ }^{28-30}$ can be very similar. Also, the additional cost of computation was considered, which made the use of the solvent models practically infeasible. Internal rotation treatment of low frequency modes ${ }^{31,32}$ in the transition state geometries has been previously shown to be important in some systems, in order to improve the accuracy of the rate predictions. However, self-initiation studies in spontaneous thermal polymerization of styrene ${ }^{20}$ and methyl acrylate ${ }^{10}$ using density functional theory calculations without applying internal rotation treatment have predicted rate constants that are comparable to experimental values, and verifiable by experiments, respectively. Also, large systems such as the present ones can have coupled rotations that are not well described by the existing models, so that applying the internal rotation model can increase the error in rate constant predictions instead of decreasing it. Reports ${ }^{32}$ also indicate that the cost of these calculations increases rapidly with the molecular size. In view of these, no special treatment of internal rotation was applied.

In this study, contour maps of singlet and triplet potential energy surfaces of ethyl and n-butyl acrylate are constructed to explore the Flory and Mayo mechanisms using first-principles density functional theory, ${ }^{33}$ with the B3LYP ${ }^{34,35}$ exchangecorrelation functional and $6-31 \mathrm{G}^{*}$ basis set. The formation of key intermediates such as 6-ethoxy-2-ethoxycarbonyl-3,4-dihydro-2H-pyran (EDP), 6-butoxy-2-butoxycarbonyl-3,4-dihydro2H-pyran (BDP), diethylcyclobutane-1,2-dicarboxylate (DECD), dibutylcyclobutane-1,2-dicarboxylate (DBCD), and singlet diradical $\left(\cdot \mathrm{M}_{2 \mathrm{~s}} \cdot\right)$, as well as the transition states and pathway for the formation of these intermediates, are also studied using B3LYP/6-31G*. Validation of these transition states was carried out using Møller-Plesset perturbation theory (MP2), again with 6-31G*. The spin-orbit coupling mechanism was studied using multiconfiguration self-consistent field (MCSCF) and 6-31G*. Monoradical generation via hydrogen transfer to or abstraction from the singlet diradical and triplet diradical intermediates by a third monomer is also investigated. The predicted monoradical species are matched to previously reported electrospray ionization-Fourier transform mass spectrometry (ESI-FTMS) data of spontaneously polymerized samples of ethyl and n-butyl acrylate to interpret the mechanism of initiation.

\section{Computational Methods}

Density functional theory ${ }^{33}$ (DFT) calculations on the singlet and triplet surfaces were performed using restricted open shell and unrestricted wave functions, respectively. B3LYP $34,35 / 6-31 \mathrm{G}^{*}$ was chosen as the level of theory to construct the potential energy surfaces and estimate transition states, due to its successful use in our previous study of free-radical polymerization of methyl acrylate. ${ }^{10}$ The molecular geometries of reactants, products, and transition states were optimized on the singlet and triplet surfaces. Hessian calculations were performed 


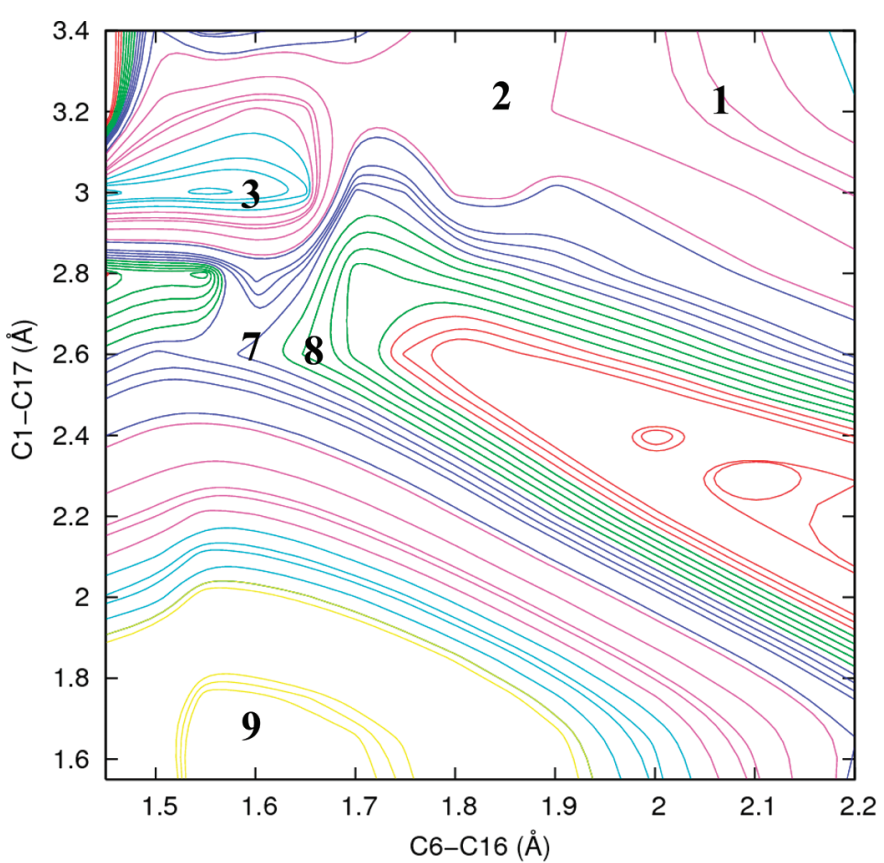

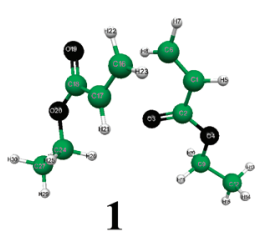

1

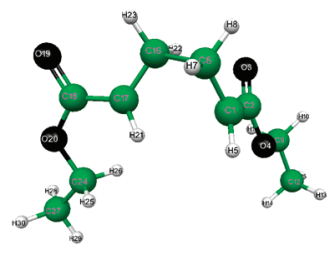

7

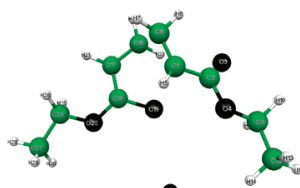

2

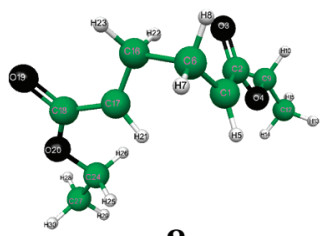

8
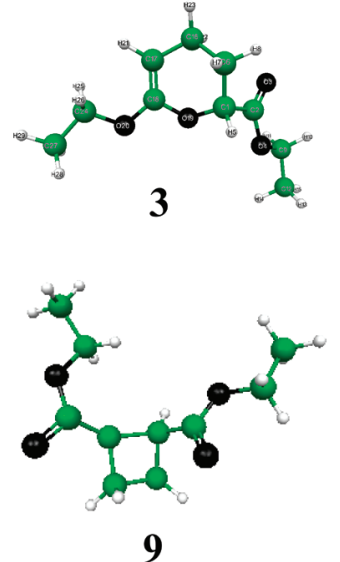

Figure 2. Contour map of the singlet potential energy surface of ethyl acrylate: $r(\mathrm{C} 1-\mathrm{C} 17)$ vs $r(\mathrm{C} 6-\mathrm{C} 16)$. All energies are relative to that of the reactant in $\mathrm{kJ} \mathrm{mol}^{-1}$. All bond lengths in $\AA$. Color scheme (highest to lowest energy): red, green, blue, magenta, cyan, yellow. The points on the low-energy pathways are connected by black lines: (1) ethyl acrylate (EA) monomers, (2) transition state (TS exo $_{\text {) }}$, (3) 6-ethoxy-2-ethoxycarbonyl3,4-dihydro-2 $\mathrm{H}$-pyran (EDP), (7) singlet diradical transition state $\left(\cdot \mathrm{M}_{2 \mathrm{~s}} \cdot\right),(\mathbf{8})$ diradical on flat region on singlet surface, (9) diethylcyclobutane1,2-diacarboxylate (DECD).

to characterize reactants and transition states. Intrinsic reaction coordinate calculations were carried out in the forward and reverse directions to determine minimum-energy pathways. Validation of the transition states and energy barriers of ethyl acrylate and n-butyl acrylate was performed with MP2/6-31G*. Spin-orbit coupling calculations ${ }^{36}$ were carried out using MCSCF/6-31G*. Reported energies (relative to the energy of the reactant) were calculated using a rigid rotor harmonic oscillator approximation (RRHO). ${ }^{37}$ Scaling factors to calculate activation entropy, temperature correction, and zero point vibrational energy in different levels of theory were taken from the National Institute of Standards and Technology (NIST) scientific and technical database. ${ }^{38}$ Rate constants were calculated using transition state theory, ${ }^{39}$ and the Wigner ${ }^{40}$ tunneling correction was used. All theoretical calculations in this work were performed using GAMESS ${ }^{41}$ in the gas phase.

\section{Results and Discussion}

3.1. Singlet Potential Energy Surface. The singlet potential energy surface of the dimerization of ethyl acrylate (EA) was constructed by constraining internuclear distances, $r(\mathrm{C} 6-\mathrm{C} 16)$ and $r(\mathrm{C} 1-\mathrm{C} 17)$, such that $1.5 \AA<r(\mathrm{C} 6-\mathrm{C} 16)<2.2 \AA$ and 1.5 $\AA<r(\mathrm{C} 1-\mathrm{C} 17) \AA<3.4 \AA$, as shown in Figure 2 . The singlet potential energy surface of the dimerization of n-butyl acrylate (nBA) was mapped by constraining internuclear distances $r(\mathrm{C} 6-\mathrm{C} 22)$ and $r(\mathrm{C} 1-\mathrm{C} 23)$ between $1.5 \AA<r(\mathrm{C} 6-\mathrm{C} 22)<2.2$ $\AA$ and $1.5 \AA<r(\mathrm{C} 1-\mathrm{C} 23)<3.4 \AA$, as shown in Figure 3 .

3.1.1. Diels-Alder Adduct Formation. Ethyl Acrylate. The $[4+2]$ cycloaddition reaction was studied by constraining bond distances, $r(\mathrm{C} 6-\mathrm{C} 16)$ and $r(\mathrm{O} 19-\mathrm{C} 1)$, in ethyl acrylate. It was found that EDP, 3, formation was predominant. The geometry of the transition state, $\mathbf{2}$, for the formation of $\mathbf{3}$ was found to have $r(\mathrm{C} 6-\mathrm{C} 16)=1.83 \AA, r(\mathrm{O} 19-\mathrm{C} 1)=2.34 \AA$, and $\Phi(\mathrm{C} 1-\mathrm{C} 6-\mathrm{C} 16-\mathrm{C} 17)=62.2^{\circ}$, and the molecular structure of 3 has $r(\mathrm{C} 6-\mathrm{C} 16)=1.53 \AA$ and $r(\mathrm{O} 19-\mathrm{C} 1)=1.53 \AA$ (Figure 2). The B3LYP/6-31G* estimated energy barrier is $139 \mathrm{~kJ}$ $\mathrm{mol}^{-1}$. IRC calculations from $\mathbf{2}$ in the forward and reverse directions show the presence of a concerted pathway. Validation studies of transition state geometry, 2, were performed with MP2/6-31G*. The calculated thermodynamic and kinetic parameters at $298 \mathrm{~K}$ are given in Table 1. 

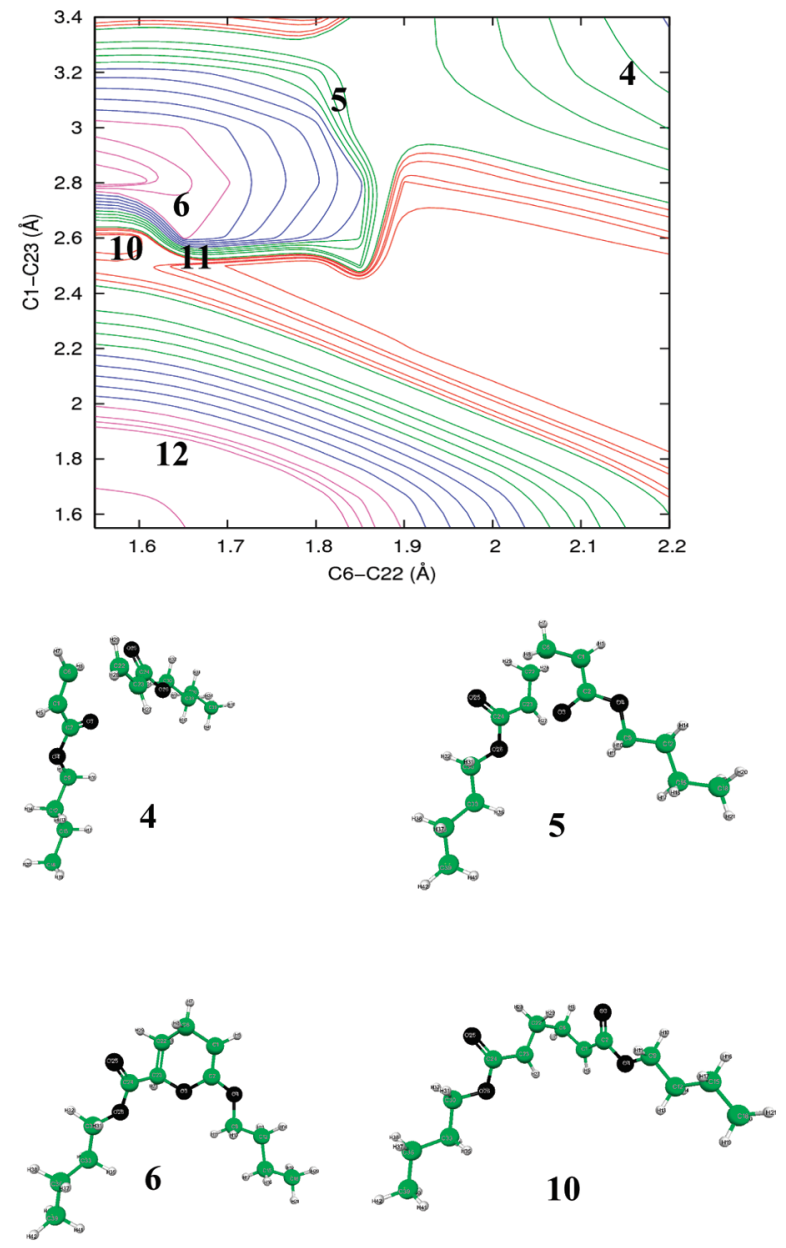

10

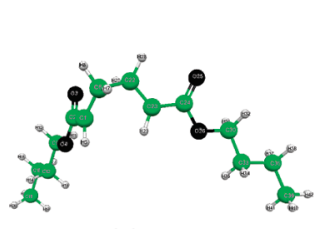

11

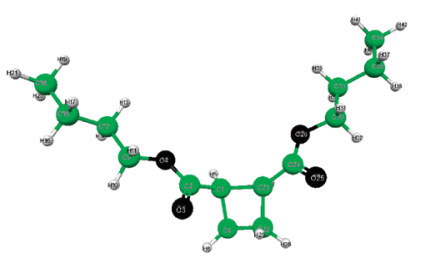

12
Figure 3. Contour map of the singlet potential energy surface of n-butyl acrylate: $r(\mathrm{C} 1-\mathrm{C} 23)$ vs $r(\mathrm{C} 6-\mathrm{C} 22)$. All energies are relative to that of the reactant in $\mathrm{kJ} \mathrm{mol}^{-1}$. All bond lengths in $\AA$. Color scheme (highest to lowest energy): red, green, blue, magenta, cyan, yellow: (4) n-butyl acrylate (nBA) monomers, $(\mathbf{5})$ transition state $\left(\mathrm{TS}_{\mathrm{exo}}\right),(\mathbf{6})$ 6-butoxy-2butoxycarbonyl-3,4-dihydro-2H-pyran (BDP), (10) singlet diradical transition state $\left(\cdot \mathrm{M}_{2 \mathrm{~s}} \cdot\right)$, (11) diradical on flat region on singlet surface, (12) dibutylcyclobutane-1,2-diacrboxylate (DBCD).

n-Butyl Acrylate. The formation of the DA dimer was studied by fixing, and subsequently relaxing, internuclear distances, $r(\mathrm{C} 6-\mathrm{C} 22)$ and $r(\mathrm{O} 3-\mathrm{C} 23)$. The transition state geometry, 5, for the formation of BDP, $\mathbf{6}$, was found to have $r(\mathrm{C} 6-\mathrm{C} 22)=$ $1.76 \AA, r(\mathrm{O} 3-\mathrm{C} 23)=2.21 \AA$, and $\Phi(\mathrm{C} 1-\mathrm{C} 6-\mathrm{C} 22-\mathrm{C} 23)=$ $63.1^{\circ}$ (Figure 3 ). The energy barrier was calculated to be $145 \mathrm{~kJ} \mathrm{~mol}^{-1}$, and IRC calculations show the presence of a concerted pathway: single transition state for bond formation between reactants and products for the formation of $\mathbf{6}$. Assessment of the transition state geometry was carried out using MP2/ 6-31G*, and the calculated enthalpies and rate constants are given in Table 1.

It was found that the standard enthalpy change for the formation of Diels-Alder adducts in ethyl and n-butyl acrylate was nearly zero $\left(\approx 1 \mathrm{~kJ} \mathrm{~mol}^{-1}\right)$. This suggests that
TABLE 1: Bond Length, Energy Barrier $\left(E_{0}\right)$, Enthalpy $\left(\Delta H^{\ddagger}{ }_{298}\right)$, and Free Energy $\left(\Delta G^{\ddagger}{ }_{298}\right)$ in $\mathrm{kJ} \mathrm{mol}^{-1}$; Frequency Factor $(A)$; and Rate Constant for DA Formation in MA, EA, n-propyl acrylate (nPA), and nBA $M^{-1} \mathrm{~s}^{-1}$ at $298 \mathrm{~K}^{a}$

\begin{tabular}{lccccrc}
\hline & $r(\mathrm{C}-\mathrm{O}) \AA$ & $r(\mathrm{C}-\mathrm{C}) \AA$ & $E_{0}$ & $\Delta H^{\ddagger}{ }_{298}$ & $\log _{\mathrm{e}} A$ & $k$ \\
\hline \multicolumn{7}{c}{ B3LYP 6-31G* } \\
MA & 2.243 & 1.773 & 122.3 & 118.41 & 8.67 & $1.41 \times 10^{-18}$ \\
EA & 2.343 & 1.83 & 139.8 & 136.2 & 9.93 & $3.78 \times 10^{-21}$ \\
nPA & 2.221 & 1.771 & 117.8 & 115 & 10.33 & $2.86 \times 10^{-17}$ \\
nBA & 2.211 & 1.767 & 145.8 & 139.9 & 6.22 & $2.1 \times 10^{-23}$ \\
\hline \multicolumn{7}{c}{ MP2 6-31G* } \\
MA & 2.009 & 1.665 & 93.9 & 89 & 7.07 & $4.07 \times 10^{-14}$ \\
EA & 2.159 & 1.635 & 112.5 & 109.1 & 10.6 & $4.12 \times 10^{-16}$ \\
nBA & 2.15 & 1.665 & 88.4 & 82.3 & 6.6 & $3.82 \times 10^{-13}$
\end{tabular}

${ }^{a}$ The reported energies are zero point vibrational energy (ZPVE) corrected.

fast equilibration of the system can lead to reversal of Diels-Alder adducts, $\mathbf{3}$ and $\mathbf{6}$, to monomers. This can also be related to the absence of $\mathrm{m} / \mathrm{z}$ peaks corresponding to Diels-Alder adducts in the ESI-FTMS spectra ${ }^{8}$ of polymer samples obtained from spontaneous solution-phase polymerization of EA and $\mathrm{nBA}$. In view of these, we suggest that DA adducts, EDP and BDP, will have a minor role in initiating polymerization of EA and $\mathrm{nBA}$.

3.1.2. DECD and DBCD Formation. A diradical mechanism for the formation of DECD, 9, and DBCD, 12, was found in thermal polymerization of $\mathrm{EA}$ and $\mathrm{nBA}$, respectively. The transition state geometry, 7, for DECD formation has $r(\mathrm{C} 6-$ $\mathrm{C} 16)=1.60 \AA, r(\mathrm{C} 1-\mathrm{C} 17)=2.77 \AA$, and $\Phi(\mathrm{C} 1-\mathrm{C} 6-\mathrm{C} 16-$ $\mathrm{C} 17)=60^{\circ}$ (Figure 2). The geometry of the diradical species, 8, has $r(\mathrm{C} 6-\mathrm{C} 16)=1.62 \AA, r(\mathrm{C} 1-\mathrm{C} 17)=2.78 \AA$, and $\Phi(\mathrm{C} 1-\mathrm{C} 6-\mathrm{C} 16-\mathrm{C} 17)=63^{\circ}$. As shown in Figure 3 , the transition state geometry, 10, for DBCD formation was found to have $r(\mathrm{C} 6-\mathrm{C} 22)=1.59 \AA, r(\mathrm{C} 1-\mathrm{C} 23)=2.81 \AA$, and $\Phi(\mathrm{C} 1-\mathrm{C} 6-\mathrm{C} 22-\mathrm{C} 23)=62.1^{\circ}$. The geometry of the singlet diradical, 11, was found to have $r(\mathrm{C} 6-\mathrm{C} 22)=1.63 \AA$, $r(\mathrm{C} 1-\mathrm{C} 23)=2.77 \AA$, and $\Phi(\mathrm{C} 1-\mathrm{C} 6-\mathrm{C} 22-\mathrm{C} 23)=65.1^{\circ}$. The calculated energy barrier using B3LYP/6-31G* for DECD formation is $189 \mathrm{~kJ} \mathrm{~mol}^{-1}$ and for DBCD formation is $198 \mathrm{~kJ}$ $\mathrm{mol}^{-1}$. IRC calculations from $\mathbf{7}$ and $\mathbf{1 0}$ showed nonconcerted pathways: one transition state for the first dimer bond formation (the diradical intermediate) and a second transition state for the formation of DECD or DBCD. Validation studies of transition state geometries for formation of DECD and DBCD were performed using MP2/6-31G*. The calculated activation energies, free energies, and rate constants are given in Table 2.

It can be seen from Table 2 that the energy barriers computed for DMCD, DECD, dipropylcyclobutane-1,2dicarboxylate (DPCD), and DBCD are similar. According to these computational results, end-substituent group has little influence on the formation of diradical species. Other cyclobutane dimers that were studied are shown in Chart 1. These dimers were found to be unstable on the singlet surface, which suggests that their formation is highly improbable.

The formation of singlet diradical in EA and nBA was found to be highly stereospecific (cis-monomer to cis-dimer and trans-monomer to trans-dimer). ${ }^{42}$ It was observed that these diradicals can undergo internal rotation and the formed cis and trans diradicals produce the cis and trans dimers, respectively. This indicates that stereorandomness, a cismonomer forming a trans-dimer and the opposite, exists in the system. A single transition state was found in the path 
TABLE 2: Bond Length, Energy Barrier $\left(E_{0}\right)$, Enthalpy $\left(\Delta H^{\dot{ }_{298}}\right)$, and Free Energy $\left(\Delta G^{\ddagger}{ }_{298}\right)$ in $\mathrm{kJ} \mathrm{mol}^{-1}$; Frequency Factor $(A)$; and Rate Constant for DMCD, DECD, DPCD, DBCD Formation in $\mathrm{M}^{-1} \mathrm{~s}^{-1}$ at $298 \mathrm{~K}$ Using Different Levels of Theory ${ }^{a}$

\begin{tabular}{lcccccc}
\hline & $r(\mathrm{C}-\mathrm{C}) \AA$ & $r(\mathrm{C}-\mathrm{C}) \AA$ & $E_{0}$ & $\Delta H^{+}{ }_{298}$ & $\log _{\mathrm{e}} A$ & $k$ \\
\hline \multicolumn{7}{c}{ B3LYP 6-31G* } \\
MA & 2.788 & 1.597 & 189.1 & 186.2 & 9.72 & $5.37 \times 10^{-30}$ \\
EA & 2.798 & 1.597 & 189 & 184.9 & 9.75 & $9.35 \times 10^{-30}$ \\
nPA & 2.789 & 1.598 & 186 & 183.4 & 10.94 & $5.58 \times 10^{-29}$ \\
nBA & 2.81 & 1.599 & 197.4 & 199 & 20.16 & $1.04 \times 10^{-27}$ \\
\hline \multicolumn{7}{c}{ MP2 6-31G* } \\
MA & 2.816 & 1.554 & 145.2 & 140.32 & 9.97 & $5.86 \times 10^{-22}$ \\
EA & 2.783 & 1.554 & 153.4 & 149.7 & 11.2 & $6.01 \times 10^{-23}$ \\
nBA & 2.80 & 1.556 & 170.8 & 167.5 & 9.68 & $9.77 \times 10^{-27}$
\end{tabular}

${ }^{a} \mathrm{DMCD}=$ dimethylcyclobutane-1,2-dicarboxylate. The reported energies are zero point vibrational energy (ZPVE) corrected.

CHART 1: (a) 3,4-Diethenyl-3,4-dimethoxy-1,2-dioxetane, (b) Dimethyl cyclobutane-1,3-dicarboxylate, and (c) Methyl-2-ethenyl-2-methoxyoxetane-3-carboxylate

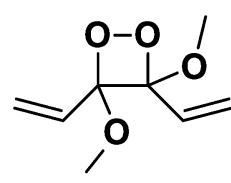

(a)

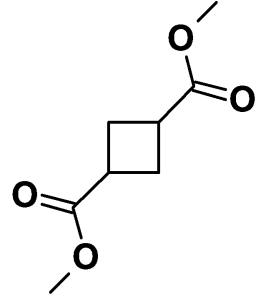

(b)

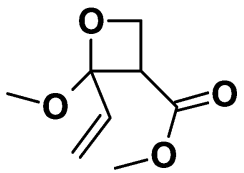

(c) for the formation of DECD and DBCD on their respective potential energy surfaces. All of these indicated that DECD and $\mathrm{DBCD}$ formation follow the one-step stereorandom mechanism proposed by Dervan and co-workers ${ }^{43}$ and is in agreement with the mechanism proposed for the formation of dimethylcyclobutane-1,2-dicarboxylate (DMCD) in spontaneous thermal polymerization of MA. ${ }^{10}$ The standard enthalpy changes for the formation of DECD and DBCD were predicted to be -60 and $-45 \mathrm{~kJ} \mathrm{~mol}^{-1}$, respectively. This indicates that the reaction is exothermic, possibly spontaneous, favoring product (DECD, DBCD) formation. However, these dimers have not been detected in solution via mass spectrometry. This points to the following possibilities: (1) DECD and DBCD may be undergoing ring-opening to form a diradical species, which then loses or gains a hydrogen upon reaction with a third monomer to form a monoradical species that initiates polymerization, (2) the singlet diradical prior to ring closure reacts with a third monomer and exchanges a hydrogen, and (3) the singlet diradical crosses over to the triplet state via the mechanism of spin-orbit coupling and the triplet diradical reacts with a third monomer to generate monoradical species. Due to very high energy barrier of the ring-opening reaction, the occurrence of this reaction at an appreciable rate is very unlikely and thus is ruled out. The occurrence of option 3 over 2 has been postulated in previous studies of spontaneous polymerization of MMA,${ }^{19}$ and our previous study showed the triplet diradical to be a key intermediate in self-initiation of methyl acrylate. ${ }^{10}$ Hence, we suggest that the formation of triplet diradical species is highly probable in thermal polymerization of EA and $\mathrm{nBA}$.

3.2. Triplet Diradical Formation. The potential energy surfaces for the formation of triplet diradical species in EA and
nBA were obtained by constraining: (for EA) $1.5 \AA<$ $r(\mathrm{C} 6-\mathrm{C} 16)<2.2 \AA$ and $1.5 \AA<r(\mathrm{C} 1-\mathrm{C} 17)<3.4 \AA$, and (for nBA) $1.5 \AA<r(\mathrm{C} 6-\mathrm{C} 22)<2.2 \AA$ and $1.5 \AA<r(\mathrm{C} 1-\mathrm{C} 23)<$ $3.4 \AA$, as shown in Figure 4. The geometry of the triplet diradical intermediate of EA, 13, has $r(\mathrm{C} 6-\mathrm{C} 16)=1.554 \AA, r(\mathrm{C} 1-\mathrm{C} 17)$ $=2.84 \AA$, and $\Phi(\mathrm{C} 1-\mathrm{C} 6-\mathrm{C} 16-\mathrm{C} 17)=64.1^{\circ}$. The geometry of the triplet diradical intermediate of nBA, 14, has $r(\mathrm{C} 6-\mathrm{C} 22)$ $=1.546 \AA, r(\mathrm{C} 1-\mathrm{C} 23)=2.845 \AA$, and $\Phi(\mathrm{C} 1-\mathrm{C} 6-\mathrm{C} 22-\mathrm{C} 23)$ $=65.1^{\circ}$. The energy of the triplet diradical intermediates of EA and nBA relative to the reactants is 128 and $110 \mathrm{~kJ} \mathrm{~mol}^{-1}$, respectively. The crossing of the singlet and triplet curves, as shown in Figure 5, indicate the occurrence of an adiabatic process. This suggests that gradually changing external conditions will allow the system to transition from an initial singlet state to a final triplet state. The spin-orbit coupling constants for the crossover of the triplet diradicals of EA and $\mathrm{nBA}$ were calculated to be 15.1 and $5.2 \mathrm{~cm}^{-1}$, respectively. The transition probability, which can be determined using the Landau-Zener model,${ }^{44}$ is directly related to the spin-orbit energy interaction matrix and subsequently the spin-orbit coupling constant. The low values of spin-orbit coupling constant correspond to low transition probability for the diradicals from the singlet to triplet state. This indicates that diradicals of EA and nBA cannot rapidly crossover from singlet to triplet. This can be attributed to the sparse manifold of vibrational states in the gas-phase dimer system, which is unfavorable for intersystem crossing. Hence, we suggest that a denser continuum of states, which would greatly accelerate singlet-triplet crossover, is most likely acquired upon collision with solvent molecules containing a heavy atom and/or inert gas molecules or nearby monomers. The increased number of states in the system enhances the probability for degeneracy of singlet-triplet vibrational states, which then leads to rapid crossover from singlet to triplet. The lack of spontaneous initiation in EA and $\mathrm{nBA}$ at lower temperatures $\left(<120^{\circ} \mathrm{C}\right)$ may be related to the reduced rate of collisions, which retards formation of a dense manifold of states and as a consequence the crossover. We conclude that, in order to obtain spontaneous polymerization of EA and nBA, high starting monomer concentrations and high temperatures are important requirements.

3.3. Monoradical Formation. The molecular assisted homolysis mechanism ${ }^{15,18}$ involving hydrogen transfer from EDP and BDP to a third monomer was studied by constraining $1.19 \AA<r(\mathrm{C} 1-\mathrm{H} 5)<1.59 \AA$ and $1.19 \AA<r(\mathrm{C} 31-\mathrm{H} 5)<1.59$ $\AA$ in EA, and $1.19 \AA<r(\mathrm{C} 1-\mathrm{H} 5)<1.59 \AA$ and $1.19 \AA<$ $r(\mathrm{C} 43-\mathrm{H} 5)<1.59 \AA$ in nBA. We found that this hydrogen transfer reaction cannot occur in EA and nBA. This is attributed to the nonaromatic nature of EDP and BDP. Since the ring structure of Diels-Alder adduct, EDP and BDP, has only one double bond (monounsaturated), it cannot provide electron delocalization after the releasing the hydrogen to the third monomer. Therefore, no stable Diels-Alder adduct and monoradical based from this adduct can be formed. This is in good agreement with results reported in thermal polymerization of $\mathrm{MMA}^{19}$ and MA. ${ }^{10}$

Hydrogen abstraction by the singlet and triplet diradical species from a third monomer and hydrogen abstraction by a third monomer from the singlet and triplet diradical species were studied. Hydrogen abstraction by the singlet and triplet diradicals from a third monomer was explored between $1.19 \AA<$ $r(\mathrm{C} 17-\mathrm{H} 31)<1.59 \AA$ and $1.19 \AA<r(\mathrm{C} 32-\mathrm{H} 31)<1.59 \AA$ in $\mathrm{EA}$, and between $1.19 \AA<r(\mathrm{C} 23-\mathrm{H} 43)<1.59 \AA$ and $1.19 \AA$ $<r(\mathrm{C} 44-\mathrm{H} 43)<1.59 \AA$ in nBA. Hydrogen transfer from the singlet and triplet diradicals to a third monomer was studied 

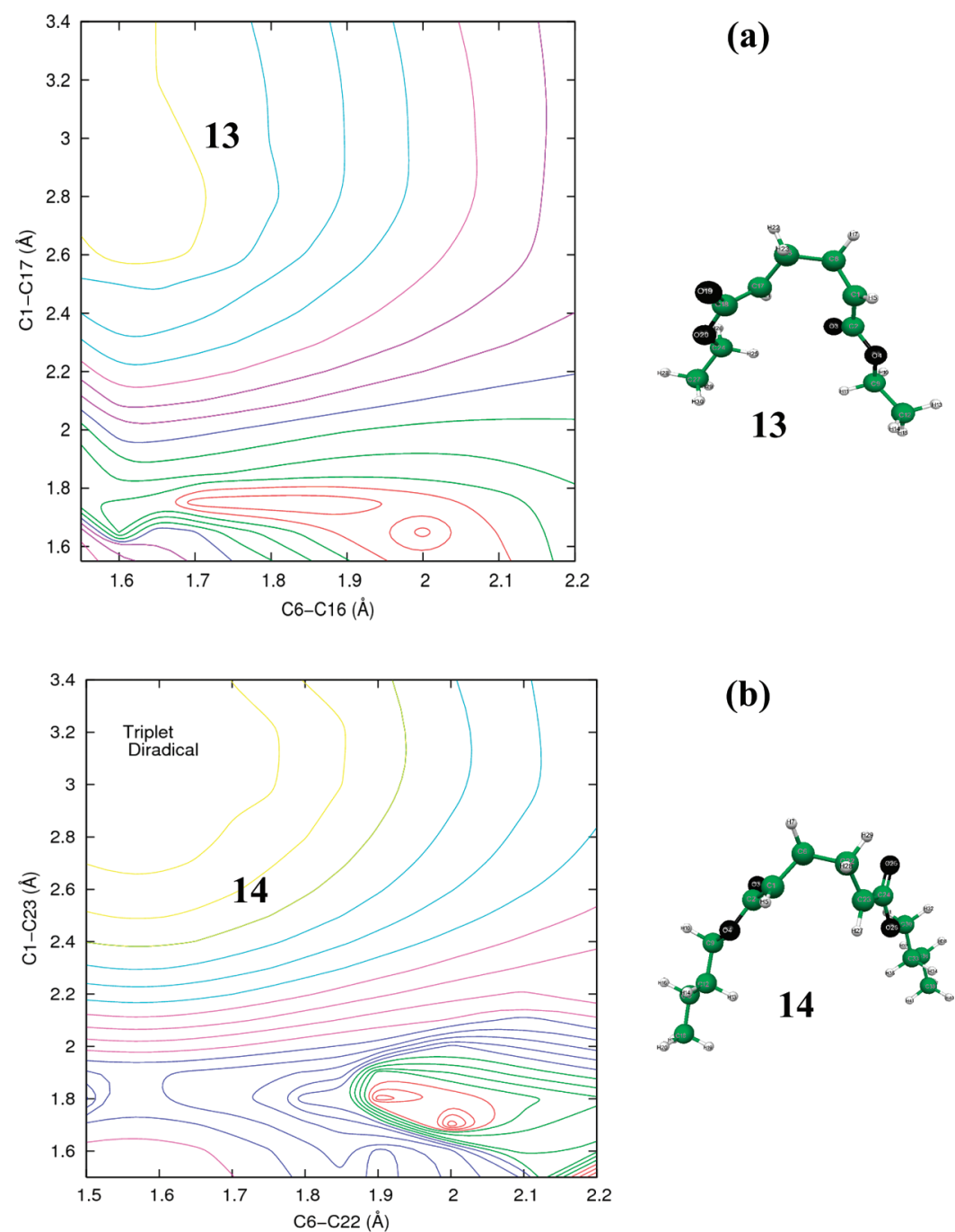

(b)

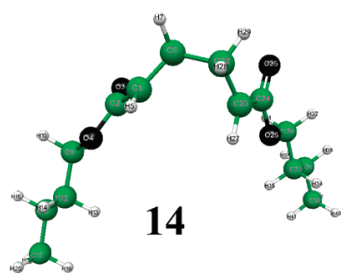

Figure 4. (a) Contour map of the triplet potential energy surface of ethyl acrylate: $r(\mathrm{C} 1-\mathrm{C} 17)$ vs $r$ (C6-C16). All energies are relative to the singlet reactant in $\mathrm{kJ} \mathrm{mol}^{-1}$. All bond lengths in $\AA$. Color nomenclature (highest to lowest energy): red, green, blue, magenta, light blue, yellow: (13) triplet diradical intermediate. (b) Contour map of the triplet potential energy surface of n-butyl acrylate: $r$ (C1-C23) vs $r$ (C6-C22). All energies are relative to the singlet reactant in $\mathrm{kJ} \mathrm{mol}^{-1}$. All bond lengths in $\AA$. Color nomenclature (highest to lowest energy): red, green, blue, magenta, light blue, yellow: (14) triplet diradical intermediate.

between $1.19 \AA<r(\mathrm{C} 6-\mathrm{H} 7)<1.59 \AA$ and $1.19 \AA<r(\mathrm{C} 31-\mathrm{H} 7)$ $<1.59 \AA$ in EA, and between $1.19 \AA<r(\mathrm{C} 6-\mathrm{H} 7)<1.59 \AA$ and $1.19 \AA<r(\mathrm{C} 43-\mathrm{H} 7)<1.59 \AA$ in $\mathrm{nBA}$. We found the ring closure of the singlet diradical in EA and nBA was preferred over hydrogen transfer and abstraction reactions. This indicates that the singlet diradical is incapable of generating monoradical species, which is comparable to the behavior of the singlet diradical in thermal polymerization of methyl acrylate. ${ }^{10}$

The triplet diradical was found to be capable of carrying out hydrogen transfer and abstraction reactions in EA and nBA. The calculated transition states for these reactions are shown in Figure 6, and the energy barriers and rate constants for initiation are given in Tables 3 and 4 . We found that the hydrogen transfer reaction from the triplet diradical to a third monomer had a lower energy barrier than hydrogen abstraction by the triplet diradical from the third monomer in EA and $\mathrm{nBA}$, which agrees with the reported results in methyl acrylate. ${ }^{10}$ Upon comparison of the rate of initiation via hydrogen transfer between MA,${ }^{10} \mathrm{EA}$, and nBA, no correlation was found to exist between energy barrier and rate constant of initiation with the size of the end substituent group. We suggest that generation of monoradical species in spontaneous thermal polymerization of EA and $\mathrm{nBA}$ occurs preferably via hydrogen transfer from the triplet radical to a third monomer than through hydrogen abstraction by the triplet diradical from a third monomer. These results strongly indicate that the triplet diradical is a key intermediate in the generation of initiating species in spontaneous thermal polymerization of alkyl acrylates.

3.4. Analysis of Chain Initiating Species in ESI-FTMS Mass Spectra. Quan et al. ${ }^{8}$ synthesized low molecular weight polymers of $\mathrm{EA}$ and $\mathrm{nBA}$ via spontaneous thermal polymerization and characterized them using ESI/FTMS. Formation of shorter live polymer chains was postulated to occur via a twostep chain transfer mechanism. The first step, backbiting, occurs by either intramolecular transfer of a proton from the middle of the propagating chain to the terminal radical group or intermolecular transfer of hydrogen from the middle of a propagating chain to a third monomer. Both these reactions cause formation of unstable midchain radicals, which undergo $\beta$-scission to form $\beta$-scission radicals and unsaturated oligomers.

The samples of poly(EA) and poly(nBA) were made at $180{ }^{\circ} \mathrm{C}$ using 40 wt $\%$ EA and nBA monomer in a mixture of o-, m-, or p-xylene. ${ }^{8}$ The molecular weights of EA and nBA monomers are 100.11 and $128.17 \mathrm{Da}$, respectively. The families of peaks, $m / z=423,523,623,723, \ldots$, in poly(EA) and $m / z=$ 


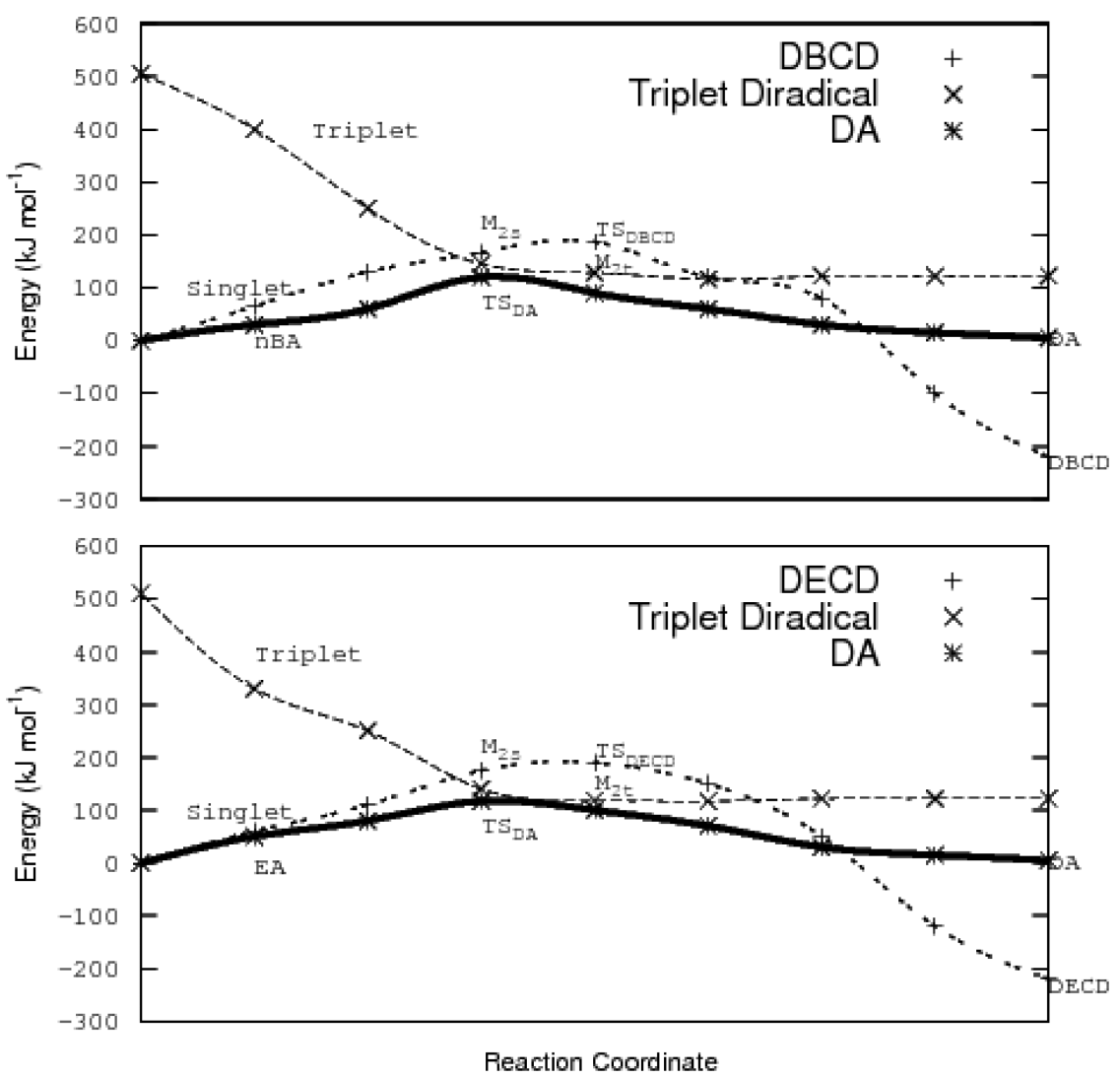

Figure 5. Reaction coordinate plot for ethyl acrylate and n-butyl acrylate.

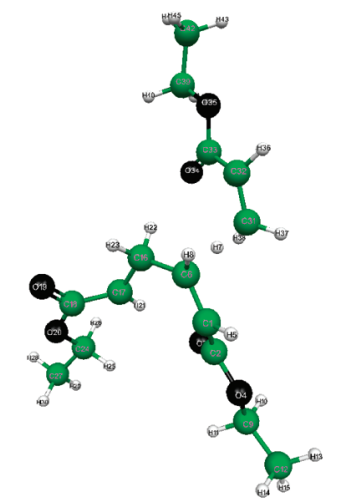

15

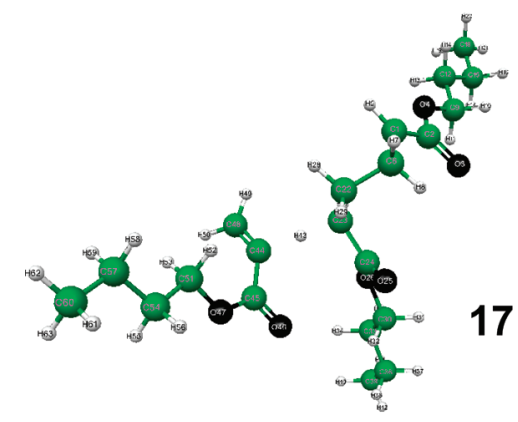

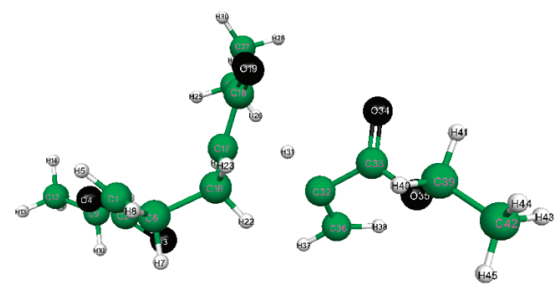

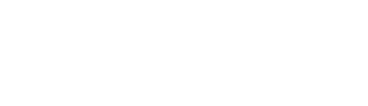

16

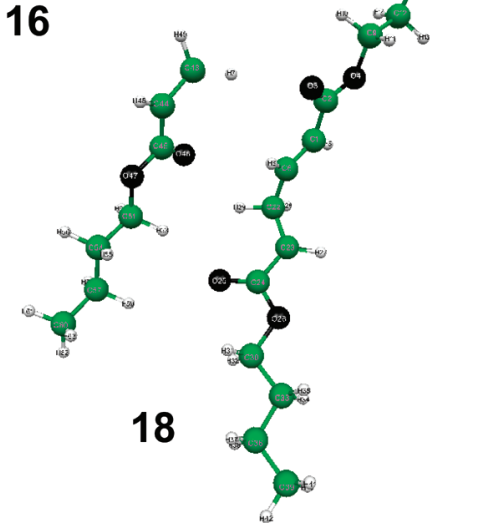

Figure 6. Transition state geometry for the formation of monoradicals in EA and nBA homopolymerizations: (16) hydrogen abstraction from a third monomer by the triplet diradical in EA polymerization, (15) hydrogen transfer from the triplet diradical to a third monomer in EA polymerization, (17) hydrogen abstraction from a third monomer by the triplet diradical in nBA polymerization, (18) hydrogen transfer from the triplet diradical to a third monomer in nBA polymerization.

$535,663,791,920, . . .$, in poly(nBA) were reported to be dominant, and the chain initiating species for these series of peaks were proposed to be $\beta$-scission radicals. ${ }^{8}$ The $m / z$ for the mass spectra is computed as a summation of initiating species 
TABLE 3: Energy Barrier $\left(E_{0}\right)$, Enthalpy $\left(\Delta H^{+}{ }_{298}\right)$, and Free Energy $\left(\Delta G^{\ddagger}{ }_{298}\right)$ in $\mathrm{kJ} \mathrm{mol}^{-1}$; Frequency Factor $(A)$; and Rate Constant for Monoradical Formation via Hydrogen Abstraction $\left(k_{\mathrm{HAE}}\right)$ and Hydrogen Transfer $\left(k_{\mathrm{TRE}}\right)$ in $\mathbf{M}^{-1} \mathbf{s}^{-1} a$

\begin{tabular}{|c|c|c|c|c|c|c|c|}
\hline$T(\mathrm{~K})$ & $E_{0}$ & $\Delta H^{\ddagger}$ & $\Delta G^{\ddagger}$ & $\log _{\mathrm{e}} A$ & $k^{b}$ without tunneling & Wigner $^{c}$ & $k^{b}$ with tunneling \\
\hline \multicolumn{8}{|c|}{ Hydrogen Transfer from $\cdot \mathrm{M}_{2 \mathrm{t}} \cdot$ to EA } \\
\hline 298 & 91.3 & 95.5 & 131.7 & 18.16 & $1.68 \times 10^{-10}$ & 4.81 & $8.09 \times 10^{-10}$ \\
\hline 373 & 91.3 & 97.7 & 140.5 & 19.29 & $6.6 \times 10^{-7}$ & 3.43 & $2.27 \times 10^{-6}$ \\
\hline \multicolumn{8}{|c|}{ Hydrogen Abstraction by $\cdot \mathrm{M}_{2 \mathrm{t}} \cdot$ from EA } \\
\hline 298 & 99.7 & 102.5 & 142.9 & 16.36 & $1.85 \times 10^{-12}$ & 2.67 & $4.9 \times 10^{-12}$ \\
\hline 373 & 99.7 & 102.6 & 151.5 & 17.28 & $1.87 \times 10^{-8}$ & 2.07 & $3.86 \times 10^{-8}$ \\
\hline
\end{tabular}

${ }^{a}$ The reported barriers are zero point vibrational energy (ZPVE) corrected. ${ }^{b}$ Rate constant from transition state theory. ${ }^{c}$ Wigner tunneling correction factor.

TABLE 4: Energy Barrier $\left(E_{0}\right)$, Enthalpy $\left(\Delta H^{\ddagger}{ }_{298}\right)$, and Free Energy $\left(\Delta G^{\ddagger}{ }_{298}\right)$ in $\mathrm{kJ} \mathrm{mol}^{-1}$; Frequency Factor $(A)$; and Rate Constant for Monoradical Formation via Hydrogen Abstraction $\left(k_{\mathrm{HAB}}\right)$ and Hydrogen Transfer $\left(k_{\mathrm{TRB}}\right)$ in $\mathbf{M}^{-1} \mathbf{s}^{-1} a$

\begin{tabular}{|c|c|c|c|c|c|c|c|}
\hline$T(\mathrm{~K})$ & $E_{0}$ & $\Delta H^{\ddagger}$ & $\Delta G^{\ddagger}$ & $\log _{\mathrm{e}} A$ & $k^{b}$ without tunneling & Wigner $^{c}$ & $k^{b}$ with tunneling \\
\hline \multicolumn{8}{|c|}{ Hydrogen Transfer from $\cdot \mathrm{M}_{2 \mathrm{t}} \cdot$ to $\mathrm{nBA}$} \\
\hline 298 & 78.1 & 79.3 & 127.02 & 13.3 & $1.11 \times 10^{-9}$ & 4.81 & $5.35 \times 10^{-9}$ \\
\hline 373 & 78.1 & 80.5 & 138.8 & 14.25 & $1.12 \times 10^{-6}$ & 3.43 & $3.85 \times 10^{-6}$ \\
\hline \multicolumn{8}{|c|}{ Hydrogen Abstraction by $\cdot \mathrm{M}_{2 \mathrm{t}} \cdot$ from nBA } \\
\hline 298 & 94.4 & 99 & 132.7 & 19.04 & $1.1 \times 10^{-10}$ & 2.67 & $2.94 \times 10^{-10}$ \\
\hline 373 & 94.4 & 101 & 141 & 20.19 & $5.63 \times 10^{-7}$ & 2.07 & $1.16 \times 10^{-6}$ \\
\hline
\end{tabular}

${ }^{a}$ The reported barriers are zero point vibrational energy (ZPVE) corrected. ${ }^{b}$ Rate constant from transition state theory. ${ }^{c}$ Wigner tunneling correction factor.

SCHEME 1: Hydrogen Transfer and Abstraction Reactions for Monoradical Generation in EA and nBA $\left(\mathrm{X}=\mathrm{C}_{2} \mathbf{H}_{5}\right.$ or $\left.\mathbf{C}_{4} \mathbf{H}_{9}\right)$

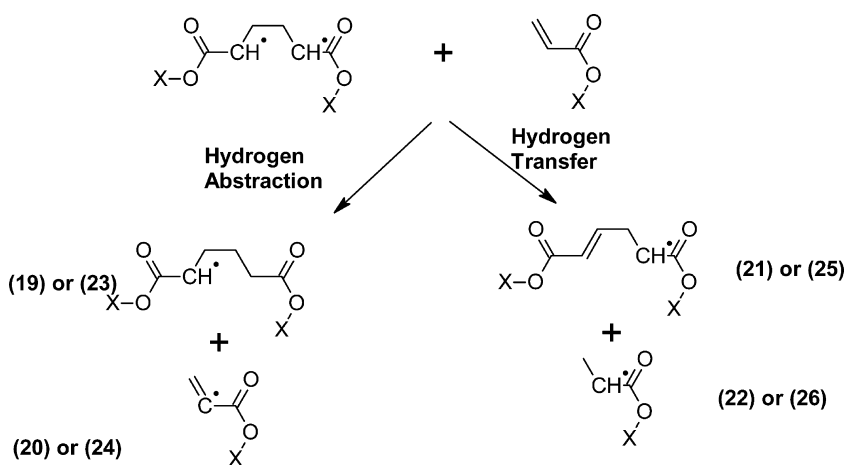

+ ion used in the spray $\left(\mathrm{Na}^{+}\right)+$number of monomers in the chain + terminating species.

We have used the monoradical species obtained via hydrogen abstraction and transfer reactions from the triplet diradical intermediate in EA and nBA (Scheme 1) in various combinations of initiating and terminating species to identify the corresponding family of peaks. The molecular weights of $\mathbf{1 9}$, 20, 21, and $\mathbf{2 2}$ are 199.22, 101.11, 201.22, and 99.11 and those of $\mathbf{2 3}, \mathbf{2 4}, \mathbf{2 5}$, and $\mathbf{2 6}$ are 255.34, 129.17, 257.34, and 127.17, respectively. It was found that the series of peaks, $\mathrm{m} / \mathrm{z}=423$, $523,623,723, \ldots$, in poly(EA) represents polymer chains initiated by 19 and terminated by 20 or vice versa, initiated by 21 and terminated by 22 or vice versa, initiated by 19 and terminated by $\mathbf{2 1}$ or vice versa, and initiated by $\mathbf{2 0}$ and terminated by 22 or vice versa. The series of peaks, $m / z=535$, $663,791,920, \ldots$, were found to represent polymer chains initiated by $\mathbf{2 3}$ and terminated by $\mathbf{2 4}$ or vice versa, initiated by $\mathbf{2 5}$ and terminated by $\mathbf{2 6}$ or vice versa, initiated by $\mathbf{2 3}$ and terminated by 25 or vice versa, and initiated by $\mathbf{2 4}$ and terminated by $\mathbf{2 6}$ or vice versa. This indicates that the calculated monoradical species are involved as chain initiating species in spontaneous thermal polymerization of EA and nBA. We recognize that such an analysis is rather qualitative, but find it sufficient to identify the initiating species by relating the calculated molecular structures and the reported mass spectra of EA and nBA.

\section{Conclusions}

The singlet and triplet potential energy surfaces for EA and nBA were mapped to study Flory and Mayo mechanisms of self-initiation. Two dimers on the singlet surface were found for both the systems. The formation of EDP and BDP was found to occur via a concerted pathway, DECD and DBCD formation via a nonconcerted pathway was identified for $\mathrm{EA}$ and $\mathrm{nBA}$, respectively. The energy barriers for the DMCD, DECD, DPCD and $\mathrm{DBCD}$ formation were found to be similar, which pointed to the lack of end substituent group effect on diradical species formation. A triplet diradical intermediate was observed in both monomers, and the spin-orbit coupling constant was calculated using MCSCF/6-31G* in EA and nBA. Monoradical generation in both systems was predicted to occur via hydrogen transfer from the triplet diradical intermediate to a third monomer, which is in good agreement with the previously observed behavior in MA. The mass of the predicted structures of the monoradicals in $\mathrm{EA}$ and $\mathrm{nBA}$ were found to correlate to the mass of chain initiating groups of the dominant series of peaks in the reported ESI/FTMS spectra of spontaneously polymerized poly(EA) and poly(nBA). Hence, we postulate that the diradical self-initiation is the most likely mechanism of initiation in spontaneous thermal polymerization of alkyl acrylates.

Acknowledgment. S.S. and M.S. acknowledge financial support from the National Science Foundation (NSF) through Grant CBET-0932882. Acknowledgment is also made to the Donors of the American Chemical Society Petroleum Research 
Fund, for partial support of this research. A.M.R. was supported by the NSF through Grant CBET-0932786, and M.W.L. by the U.S. Air Force Office of Scientific Research, under Grant FA 9550-10-1-0248. Computational support was provided by the High-Performance Computing Modernization Office of the U.S. Department of Defense.

\section{References and Notes}

(1) Adamsons, K.; Blackman, G.; Gregorovich, B.; Lin, L.; Matheson, R. Prog. Org. Coat. 1998, 34, 64 .

(2) VOC's Directive; EU Committee of the American Chamber of Commerce in Belgium, ASBL/VZw, Brussels, July 8, 1996.

(3) Chiefari, J.; Jeffery, J.; Mayadunne, R. T. A.; Moad, G.; Rizzardo, E.; Thang, S. H. Macromolecules 1999, 32, 7700-7702.

(4) Grady, M. C.; Simonsick, W. J.; Hutchinson, R. A. Macromol. Symp. 2002, 182, 149-168.

(5) Peck, A. N. F.; Hutchinson, R. A.; Grady, M. C. Polym. Prepr. 2002, 43, 154-155. 5951.

(6) Peck, A. N. F.; Hutchinson, R. A. Macromolecules 2004, 37, 5944-

(7) Grady, M. C.; Quan, C.; Soroush, M. Patent Application Number 60/484,393, filed on July 2, 2003.

(8) Quan, C.; Soroush, M.; Grady, M. C.; Hansen, J. E.; Simonsick, W. J. Macromolecules 2005, 38, 7619-7628.

(9) Junkers, T.; Bennet, F.; Koo, S. S.; Barner-Kowollik, B. J. Polym. Sci. 2008, 46, 3433-3437.

(10) Srinivasan, S.; Lee, M. W.; Grady, M. C.; Soroush, M; Rappe, A. M. J. Phys. Chem. A 2009, 110, 10787-10794.

(11) Srinivasan, S. Ph.D. Thesis. Drexel University, Philadelphia, PA, 2009.

(12) Walling, C.; Briggs, E. R.; Mayo, F. R. J. Am. Chem. Soc. 1946, $68,1145-1149$.

(13) Flory, P. J. J. Am. Chem. Soc. 1937, 59, 241-253.

(14) Mayo, F. R. J. Am. Chem. Soc. 1953, 75, 6133-6142.

(15) Hiatt, R. R.; Bartlett, P. D. J. Am. Chem. Soc. 1959, 81, 11491154.

(16) Mayo, F. R. J. Am. Chem. Soc. 1968, 90, 1289-1295.

(17) Buzanowski, W. C.; Graham, J. D.; Priddy, D. B.; Shero, E. Polymer 1992, 33, 3055-3059.

(18) Pryor, W. A.; Lasswell, L. D. Adv. Free-Radical Chem. 1975, 5, 27-99.

(19) Lingnau, J.; Stickler, M.; Meyerhoff, G. Eur. Polym. J. 1980, 16 785-791.
(20) Khuong, K. S.; Jones, W. H.; Pryor, W. A.; Houk, K. N. J. Am. Chem. Soc. 2005, 127, 1265-1277.

(21) Salem, L.; Rowland, C. Angew. Chem., Int. Ed. 1972, 11, 92-111.

(22) Robinson, G. W. J. Chem. Phys. 1967, 47, 1967-1979.

(23) Anderson, L. G.; Paramenter, C. S. J. Chem. Phys. 1970, 52, 466468.

(24) Eder, T. W.; Carr, R. W. J. Chem. Phys. 1970, 53, 2258-2266.

(25) Kropp, P. J. J. Am. Chem. Soc. 1969, 91, 5783-5791.

(26) Dunning, T. H.; Hunt, W. J.; Goddard, W. A., III. Chem. Phys. Lett. 1969, 4, 147-150.

(27) Harrison, J. F.; Allen, L. C. J. Am. Chem. Soc. 1969, 91, 807-823.

(28) Pleigo, J. R., Jr.; Riveros, J. M. J. Phys. Chem. A 2001, 105, 72417247

(29) Truhlar, D. G.; Garrett, B. C.; Klippenstein, S. J. J. Phys. Chem. 1996, 100, 12771-12800.

(30) Thickett, S. C.; Gilbert, R. G. Polymer 2004, 45, 6993-6999.

(31) Pitzer, K. S.; Gwinn, W. D. J. Chem. Phys. 1942, 10, 428-440. Truhlar, D. G. J. Comput. Chem. 1991, 12, 266-270. Ayala, P. Y.; Schlegel, H. B. J. Chem. Phys. 1998, 108, 2314-2325. Van Speybroeck, V.; Van Neck, D.; Waroquier, M.; Wauters, S.; Saeys, M.; Marin, G. B. J. Phys. Chem. A 2000, 104, 10939-10950.

(32) Pfaendtner, J.; Yu, X; Broadbelt, L. J. Theor. Chem. Acc. 2007, $118,881-898$, and references within.

(33) Hohenberg, P.; Kohn, W. Phys. Rev. 1964, 136, B864-B871.

(34) Becke, A. D. Phys. Rev. A 1988, 38, 3098-3100.

(35) Lee, C.; Yang, W.; Parr, R. G. Phys. Rev. B 1988, 37, 785-789.

(36) Fedorov, D. G.; Koseki, S.; Schmidt, M. W.; Gordon, M. S. Int. Rev. Phys. Chem. 2003, 22, 551-592.

(37) Irikura, K. K. THERMO PL; National Institute of Standards and Technology: Gaithersburg, MD, 2002.

(38) http://cccbdb.nist.gov/; accessed 06/11/2010.

(39) Eyring, H. J. Chem. Phys. 1935, 3, 107-115.

(40) Wigner, E. P. Z. Phys. Chem., Abt. B 1932, 19, 203-216.

(41) Schmidt, M. W.; Baldridge, K. K.; Boatz, J. A.; Elbert, S. T.; Gordon, M. S.; Jensen, J. H.; Koseki, S.; Matsunaga, N.; Nguyen, K. A.; Su, S. J.; Windus, T. L.; Dupuis, M.; Montgomery, J. A. J. Comput. Chem. 1993, 14, 1347.

(42) Woodward, R. B.; Hoffmann, R. J. Am. Chem. Soc. 1965, 87, 395397.

(43) Dervan, P.; Santilli, D. J. J. Am. Chem. Soc. 1980, 102, 38633870

(44) Landau, L. Phys. Sov. U. 1932, 2, 46-51. Zener, C. Proc. R. Soc. London, Ser. A 1932, 137, 692-702.

JP102772V 